\author{
E. A. Sevost'yanov, S. A. Skvortsov, N. S. Ilkevych
}

\title{
ON BOUNDARY BEHAVIOR OF MAPPINGS WITH TWO NORMALIZED CONDITIONS
}

\begin{abstract}
E. A. Sevost'yanov, S. A. Skvortsov, N. S. Ilkevych. On boundary behavior of mappings with two normalized conditions, Mat. Stud. 49 (2018), 150-157.

The paper is devoted to a study of mappings with finite distortion that have been recently actively investigated last time. We study the boundary behavior of mappings between two fixed domains in metric spaces, which satisfy some moduli estimates. We have proved that families of corresponding inverse mappings with two normalized conditions and integrable majorant are equicontinuous whenever the domain of the mappings has a weakly flat boundary.
\end{abstract}

1. Introduction. In our recent paper [1], we have obtained equicontinuity of classes of mappings between two domains of metric spaces $(X, d)$ and $\left(X^{\prime}, d^{\prime}\right)$, for which inverse of the mappings satisfy some modulus inequality. The equicontinuity of those classes in $\overline{D^{\prime}}$, consisting of mappings $g=f^{-1}$ of $D^{\prime} \subset X^{\prime}$ onto $D \subset X$, is established under two main conditions, one of which is

$$
\operatorname{diam} f(A):=\sup _{x, y \in A} d(x, y) \geqslant \delta>0,
$$

where $A \subset D$ is a fixed continuum, and $\delta>0$ does not depend on $f$. The second condition is a requirement that $\partial D^{\prime}$ does not contain any non-degenerate continuum. Remark that, this condition seems us too strong, and we will try to replace it by another assumptions. The main goal of the present paper is to prove some results of [1] under weaker conditions on $\partial D^{\prime}$. Results given below are new even in Euclidean case, however, they due to general metric spaces. For notions and definitions used below we refer reader to [1], cf. [2] and [3].

Recall, for a given continuous path $\gamma:[a, b] \rightarrow X$ in a metric space $(X, d)$, that its length is the supremum of the sums

$$
\sum_{i=1}^{k} d\left(\gamma\left(t_{i}\right), \gamma\left(t_{i-1}\right)\right)
$$

over all partitions $a=t_{0} \leqslant t_{1} \leqslant \ldots \leqslant t_{k}=b$ of the interval $[a, b]$. The path $\gamma$ is called rectifiable if its length is finite. Given a family of paths $\Gamma$ in $X$, a Borel function $\rho: X \rightarrow[0, \infty]$ is called admissible for $\Gamma$, abbr. $\rho \in \operatorname{adm} \Gamma$, if

$$
\int_{\gamma} \rho d s \geqslant 1
$$

2010 Mathematics Subject Classification: 30C65, 30L10, 31C12.

Keywords: metric spaces; quasiconformal mappings; mappings with bounded and finite distortion; equicontinuity; moduli of families of paths.

doi:10.15330/ms.49.2.150-157

(C) E. A. Sevost'yanov, S. A. Skvortsov, N. S. Ilkevych, 2018 
for all (locally rectifiable) $\gamma \in \Gamma$. In what follows, $\operatorname{adm} \Gamma$ is the set of all admissible functions for $\Gamma$. Everywhere further, for any sets $E, F$, and $G$ in $X$, we denote by $\Gamma(E, F, G)$ the family of all continuous paths $\gamma:[0,1] \rightarrow X$ such that $\gamma(0) \in E, \gamma(1) \in F$, and $\gamma(t) \in G$ for all $t \in(0,1)$. Everywhere further $(X, d, \mu)$ and $\left(X^{\prime}, d^{\prime}, \mu^{\prime}\right)$ are metric spaces with metrics $d$ and $d^{\prime}$ and locally finite Borel measures $\mu$ and $\mu^{\prime}$, correspondingly. We will assume that $\mu$ is a Borel measure such that $0<\mu(B)<\infty$ for all balls $B$ in $X$. Given $p \geqslant 1$, the $p$-modulus of the family $\Gamma$ is the number

$$
M_{p}(\Gamma)=\inf _{\rho \in \operatorname{adm} \Gamma} \int_{X} \rho^{p}(x) d \mu(x) .
$$

Should adm $\Gamma$ be empty, we set $M_{p}(\Gamma)=\infty$. Let $G$ and $G^{\prime}$ be domains with finite Hausdorff dimensions $\alpha$ and $\alpha^{\prime} \geqslant 1$ in spaces $(X, d, \mu)$ and $\left(X^{\prime}, d^{\prime}, \mu^{\prime}\right)$, and let $Q: G \rightarrow[0, \infty]$ be a measurable function. Given $x_{0} \in \partial G$, denote $S_{i}:=S\left(x_{0}, r_{i}\right)=\left\{x \in G: d\left(x, x_{0}\right)=r_{i}\right\}$, $i=1,2$, where $0<r_{1}<r_{2}<\infty$. We say that a mapping $f: G \rightarrow G^{\prime}$ is a ring $Q$-mapping at a point $x_{0} \in \bar{G}$, if the inequality

$$
M_{\alpha^{\prime}}\left(f\left(\Gamma\left(S_{1}, S_{2}, A\right)\right)\right) \leqslant \int_{A \cap G} Q(x) \eta^{\alpha}\left(d\left(x, x_{0}\right)\right) d \mu(x)
$$

holds for any ring

$$
A=A\left(x_{0}, r_{1}, r_{2}\right)=\left\{x \in X: r_{1}<d\left(x, x_{0}\right)<r_{2}\right\}, \quad 0<r_{1}<r_{2}<\infty,
$$

and any measurable function $\eta:\left(r_{1}, r_{2}\right) \rightarrow[0, \infty]$ such that

$$
\int_{r_{1}}^{r_{2}} \eta(r) d r \geqslant 1
$$

holds. We say that $f$ is a ring $Q$-homeomorphism in $E \subset \bar{D}$, if and only if $f$ is a ring $Q$-homeomorphism for every $x_{0} \in E$.

We say that the boundary of $D$ is weakly flat at a point $x_{0} \in \partial D$ if for every number $P>0$ and every neighborhood $U$ of the point $x_{0}$ there is a neighborhood $V \subset U$ such that $M_{\alpha}(\Gamma(E, F, D)) \geqslant P$ for all continua $E$ and $F$ in $D$ intersecting $\partial U$ and $\partial V$. We say that the boundary $\partial D$ is weakly flat if the corresponding property holds at every point of the boundary.

In what follows,

$$
|\gamma|:=\{x \in X: \exists t \in[a, b]: \gamma(t)=x\}
$$

is a locus of a path $\gamma:[a, b] \rightarrow X$. Two paths $C_{1}:[a, b] \rightarrow X$ and $C_{2}:[a, b] \rightarrow X$ are called disjoint if and only if $\left|C_{1}\right| \cap\left|C_{2}\right|=\varnothing$. In what follows, we consider the following condition $\mathbf{A}$ :

$\boldsymbol{A} \Leftrightarrow$ any pair of points $a \in D, b \in \bar{D}$, and $c \in D, d \in \bar{D}$ can be joined by disjoint paths $C_{1}$ and $C_{2}$ in $D$.

We have proved that domains in $\mathbb{R}^{n}, n \geqslant 2$, which are locally connected on the boundary, satisfy the condition A, see [1, Proposition]. 
Proposition 1. Let $D$ be a domain in $\mathbb{R}^{n}, n \geqslant 2$, which is locally connected on the boundary. Then any two pairs of points $a \in D, b \in \bar{D}$, and $c \in D, d \in \bar{D}$ can by joined by disjoint paths $\gamma_{1}:[0,1] \rightarrow \bar{D}$ and $\gamma_{2}:[0,1] \rightarrow \bar{D}$, such that $\gamma_{i}(t) \in D$ for all $t \in(0,1), i=1,2, \gamma_{1}(0)=a$, $\gamma_{1}(1)=b, \gamma_{2}(0)=c, \gamma_{2}(1)=d$.

A domain $D$ is said to be locally (path) connected at $x_{0} \in \bar{D}$, if for every neighborhood $U$ of $x_{0}$ there exists a neighborhood $V \subset U$ of $x_{0}$ such that $V \cap D$ is (path) connected. A domain $D$ is locally (path) connected in $E \subset \bar{D}$, if the corresponding property holds for every $x_{0} \in E$.

Given domains $D \subset X, D^{\prime} \subset X^{\prime}, w_{1}, w_{2} \in D, w_{1}^{\prime}, w_{2}^{\prime} \in D^{\prime}$, and a measurable function $Q: X \rightarrow[0, \infty], Q(x) \equiv 0$ for $x \notin D$, denote by $\mathfrak{R}_{Q}^{w_{1}, w_{2}, w_{1}^{\prime}, w_{2}^{\prime}}\left(D, D^{\prime}\right)$ a family of all homeomorphisms $g$ of $D^{\prime}$ onto $D$ such that $f=g^{-1}, f: D \rightarrow D^{\prime}$, is a ring $Q$-homeomorphism in $\bar{D}$ with $f\left(w_{1}\right)=w_{1}^{\prime}, f\left(w_{2}\right)=w_{2}^{\prime}$. The following result holds.

Theorem 1. Let $D \subset X$ and $D^{\prime} \subset X^{\prime}$ be domains with Hausdorff dimensions $\alpha \geqslant 2$ and $\alpha^{\prime} \geqslant 2$, correspondingly. Suppose that:

1) $D$ is locally path connected on $\bar{D}$;

2) $\bar{D}$ and $\overline{D^{\prime}}$ are compacts in $X$ and $X^{\prime}$, correspondingly;

3) $D^{\prime}$ has a weakly flat boundary;

4) the condition $\boldsymbol{A}$ holds;

5) $Q \in L^{1}(D)$.

Now, every $g \in \mathfrak{R}_{Q}^{w_{1}, w_{2}, w_{1}^{\prime}, w_{2}^{\prime}}\left(D, D^{\prime}\right)$ has a continuous extension on $\partial D^{\prime}$ such that $g\left(\overline{D^{\prime}}\right)=\bar{D}$, and the family $\mathfrak{R}_{Q}^{w_{1}, w_{2}, w_{1}^{\prime}, w_{2}^{\prime}}\left(\bar{D}, \overline{D^{\prime}}\right)$, consisting of all extended mappings $\bar{g}: \overline{D^{\prime}} \rightarrow \bar{D}$, is equicontinuous on $\partial D^{\prime}$.

Given domains $D, D^{\prime} \subset \mathbb{R}^{n}, n \geqslant 2, w_{1}, w_{2} \in D, w_{1}^{\prime}, w_{2}^{\prime} \in D^{\prime}$, and a Lebesgue measurable function $Q: \mathbb{R}^{n} \rightarrow[0, \infty], Q(x) \equiv 0$ for $x \notin D$, denote by $\mathfrak{E}_{Q}^{w_{1}, w_{2}, w_{1}^{\prime}, w_{2}^{\prime}}\left(D, D^{\prime}\right)$ a family of all homeomorphisms $g: D^{\prime} \rightarrow D$ of $D^{\prime}$ onto $D$ such that $f=g^{-1}, f: D \rightarrow D^{\prime}$, is a ring $Q$-homeomorphism in $\bar{D}$ with $f\left(w_{1}\right)=w_{1}^{\prime}, f\left(w_{2}\right)=w_{2}^{\prime}$. Since the condition A holds in $\mathbb{R}^{n}$ for corresponding domains, see Proposition 1 , we obtain the following consequence from Theorem 1.

Corollary 1. Let $D, D^{\prime} \subset \mathbb{R}^{n}, n \geqslant 2$, be domains with compact closure in $\mathbb{R}^{n}$. Assume that $D$ is locally path connected on $\bar{D}, D^{\prime}$ has a weakly flat boundary and $Q \in L^{1}(D)$.

Now, every $g \in \mathfrak{E}_{Q}^{w_{1}, w_{2}, w_{1}^{\prime}, w_{2}^{\prime}}\left(D, D^{\prime}\right)$ has a continuous extension on $\partial D^{\prime}$ such that $g\left(\overline{D^{\prime}}\right)=$ $\bar{D}$, and the family $\mathfrak{E}_{Q}^{w_{1}, w_{2}, w_{1}^{\prime}, w_{2}^{\prime}}\left(\bar{D}, \overline{D^{\prime}}\right)$, consisting of all extended mappings $\bar{g}: \overline{D^{\prime}} \rightarrow \bar{D}$, is equicontinuous on $\partial D^{\prime}$.

\section{Proof of Theorem 1.}

Proof of Theorem 1. Let $g \in \mathfrak{R}_{Q}^{w_{1}, w_{2}, w_{1}^{\prime}, w_{2}^{\prime}}\left(D, D^{\prime}\right)$. Since $D^{\prime}$ has a weakly flat boundary, $g$ extends to a continuous mapping $\bar{g}: \overline{D^{\prime}} \rightarrow \bar{D}$ (see [4, Theorem 3], cf. [2, Theorem 4.6] and [3, Theorem 6.1]).

Let us to verify the equality $\bar{g}\left(\overline{D^{\prime}}\right)=\bar{D}$. Indeed, by definition, $\bar{g}\left(\overline{D^{\prime}}\right) \subset \bar{D}$. It remains to show the converse inclusion $\bar{D} \subset \bar{g}\left(\overline{D^{\prime}}\right)$. Let $x_{0} \in \bar{D}$. Now, we show that $x_{0} \in \bar{g}\left(\overline{D^{\prime}}\right)$. If 
$x_{0} \in \bar{D}$, then either $x_{0} \in D$, or $x_{0} \in \partial D$. If $x_{0} \in D$, then there is nothing to prove, since by hypothesis $\bar{g}\left(D^{\prime}\right)=D$. Let $x_{0} \in \partial D$. Now, there exist $x_{k} \in D$ and $y_{k} \in D^{\prime}$ such that $x_{k}=\bar{g}\left(y_{k}\right)$ and $x_{k} \rightarrow x_{0}$ as $k \rightarrow \infty$. Since $\overline{D^{\prime}}$ is compact, we may assume that $y_{k} \rightarrow y_{0} \in \overline{D^{\prime}}$ as $k \rightarrow \infty$. Since $f=g^{-1}$ is a homeomorphism, $y_{0} \in \partial D^{\prime}$. Since $\bar{g}^{-1}$ is continuous in $\overline{D^{\prime}}$, $\bar{g}\left(y_{k}\right) \rightarrow \bar{g}\left(y_{0}\right)$. However, in this case, $\bar{g}\left(y_{0}\right)=x_{0}$, because $\bar{g}\left(y_{k}\right)=x_{k}$ and $x_{k} \rightarrow x_{0}$ as $k \rightarrow \infty$. Thus, $x_{0} \in \bar{g}\left(\overline{D^{\prime}}\right)$. The inclusion $\bar{D} \subset \bar{g}\left(\overline{D^{\prime}}\right)$ is proved. Therefore, $\bar{D}=\bar{g}\left(\overline{D^{\prime}}\right)$, as required.

It remains to show that $\mathfrak{R}_{Q}^{w_{1}, w_{2}, w_{1}^{\prime}, w_{2}^{\prime}}\left(\bar{D}, \overline{D^{\prime}}\right)$ is equicontinuous at $\partial D^{\prime}$. We give the proof by contradiction. Now, we can find a point $z_{0} \in \partial D^{\prime}$, a number $\varepsilon_{0}>0$ and sequences $z_{m} \in \overline{D^{\prime}}, z_{m} \rightarrow z_{0}$ as $m \rightarrow \infty$ and $\bar{g}_{m} \in \mathfrak{R}_{Q}^{w_{1}, w_{2}, w_{1}^{\prime}, w_{2}^{\prime}}\left(\bar{D}, \overline{D^{\prime}}\right)$ such that

$$
d\left(\bar{g}_{m}\left(z_{m}\right), \bar{g}_{m}\left(z_{0}\right)\right) \geqslant \varepsilon_{0}, \quad m=1,2, \ldots
$$

Put $g_{m}:=\left.\bar{g}_{m}\right|_{D^{\prime}}$. Since $g_{m}$ has a continuous extension on $\partial D^{\prime}$, we may assume that $z_{m} \in D^{\prime}$ and $\bar{g}_{m}\left(z_{m}\right)=g_{m}\left(z_{m}\right)$. In addition, there exists $z_{m}^{\prime} \in D^{\prime}, z_{m}^{\prime} \rightarrow z_{0}$ as $m \rightarrow \infty$, such that $d\left(g_{m}\left(z_{m}^{\prime}\right), \bar{g}_{m}\left(z_{0}\right)\right) \rightarrow 0$ as $m \rightarrow \infty$. Since $\bar{D}$ is compact, we may assume that $g_{m}\left(z_{m}\right)$ and $\bar{g}_{m}\left(z_{0}\right)$ have limits as $m \rightarrow \infty$. Let $g_{m}\left(z_{m}\right) \rightarrow \overline{x_{1}}$ and $\bar{g}_{m}\left(z_{0}\right) \rightarrow \overline{x_{2}}$ as $m \rightarrow \infty$. By continuity of the metrics in (4), $\overline{x_{1}} \neq \overline{x_{2}}$. Since homeomorphisms preserve a boundary, $\overline{x_{2}} \in \partial D$. Without loss of generality, we may assume that $w_{1} \neq \overline{x_{1}}$. By the condition $\mathbf{A}$, we may join points $w_{1}$ and $\overline{x_{1}}$ by the path $\gamma_{1}:[0,1] \rightarrow \bar{D}$, and points $w_{2}$ and $\overline{x_{2}}$ by the path $\gamma_{2}:[0,1] \rightarrow \bar{D}$ such that $\left|\gamma_{1}\right| \cap\left|\gamma_{2}\right|=\varnothing, \gamma_{i}(t) \in D$ for all $t \in(0,1), i=1,2, \gamma_{1}(0)=w_{1}, \gamma_{1}(1)=\overline{x_{1}}, \gamma_{2}(0)=w_{2}$ and $\gamma_{2}(1)=\overline{x_{2}}$. Since $D$ is locally connected on $\partial D$, there are neighborhoods $U_{1}$ and $U_{2}$ of $\overline{x_{1}}$ and $\overline{x_{2}}$, whose closures do not intersect, and $W_{i}:=D \cap U_{i}$ are path-connected sets. In what follows,

$$
B\left(x_{0}, r\right):=\left\{x \in X: d\left(x, x_{0}\right)<r\right\} .
$$

Without loss of generality, we may assume that $\overline{U_{1}} \subset B\left(\overline{x_{1}}, \delta_{0}\right)$ and

$$
\overline{B\left(\overline{x_{1}}, \delta_{0}\right)} \cap\left|\gamma_{2}\right|=\varnothing=\overline{U_{2}} \cap\left|\gamma_{1}\right|, \quad \overline{B\left(\overline{x_{1}}, \delta_{0}\right)} \cap \overline{U_{2}}=\varnothing,
$$

$g_{m}\left(z_{m}\right) \in W_{1}$ and $g_{m}\left(z_{m}^{\prime}\right) \in W_{2}$ for each $m \in \mathbb{N}$. Let $a_{1}$ and $a_{2}$ be arbitrary points in $\left|\gamma_{1}\right| \cap W_{1}$ and $\left|\gamma_{2}\right| \cap W_{2}$, correspondingly. Let $t_{1}, t_{2}$ be such that $\gamma_{1}\left(t_{1}\right)=a_{1}$ and $\gamma_{2}\left(t_{2}\right)=a_{2}$. We join $a_{1}$ and $g_{m}\left(z_{m}\right)$ by a path $\alpha_{m}:\left[t_{1}, 1\right] \rightarrow W_{1}$ such that $\alpha_{m}\left(t_{1}\right)=a_{1}$ and $\alpha_{m}(1)=g_{m}\left(z_{m}\right)$. Similarly, we join $a_{2}$ and $g_{m}\left(z_{m}^{\prime}\right)$ by a path $\beta_{m}:\left[t_{2}, 1\right] \rightarrow W_{2}, \beta_{m}\left(t_{2}\right)=a_{2}$ and $\beta_{m}(1)=g_{m}\left(z_{m}^{\prime}\right)$, see Figure 1.

Set

$$
C_{m}^{1}(t)=\left\{\begin{array}{ll}
\gamma_{1}(t), & t \in\left[0, t_{1}\right], \\
\alpha_{m}(t), & t \in\left[t_{1}, 1\right]
\end{array}, \quad C_{m}^{2}(t)=\left\{\begin{array}{ll}
\gamma_{2}(t), & t \in\left[0, t_{2}\right], \\
\beta_{m}(t), & t \in\left[t_{2}, 1\right]
\end{array} .\right.\right.
$$

Given sets $A, B \subset X$, denote

$$
\operatorname{dist}(A, B):=\inf _{x \in A, y \in B} d(x, y) .
$$

Denote, as usual, $\left|C_{m}^{1}\right|$ and $\left|C_{m}^{2}\right|$ are loci of paths $C_{m}^{1}$ and $C_{m}^{2}$, respectively. Setting

$$
l_{0}=\min \left\{\operatorname{dist}\left(\left|\gamma_{1}\right|,\left|\gamma_{2}\right|\right), \operatorname{dist}\left(\left|\gamma_{1}\right|, U_{2}\right)\right\},
$$

we consider the covering $A_{0}:=\bigcup_{x \in\left|\gamma_{1}\right|} B\left(x, l_{0} / 4\right)$. Since $\left|\gamma_{1}\right|$ is a compact, we can choose $1 \leqslant N_{0}<\infty$ and points $x_{1}, \ldots, x_{N_{0}} \in\left|\gamma_{1}\right|$ such that $\left|\gamma_{1}\right| \subset B_{0}:=\bigcup_{i=1}^{N_{0}} B\left(x_{i}, l_{0} / 4\right)$. Now

$$
\left|C_{m}^{1}\right| \subset U_{1} \cup\left|\gamma_{1}\right| \subset \overline{B\left(\overline{x_{1}}, \delta_{0}\right)} \cup \bigcup_{i=1}^{N_{0}} B\left(x_{i}, l_{0} / 4\right) .
$$




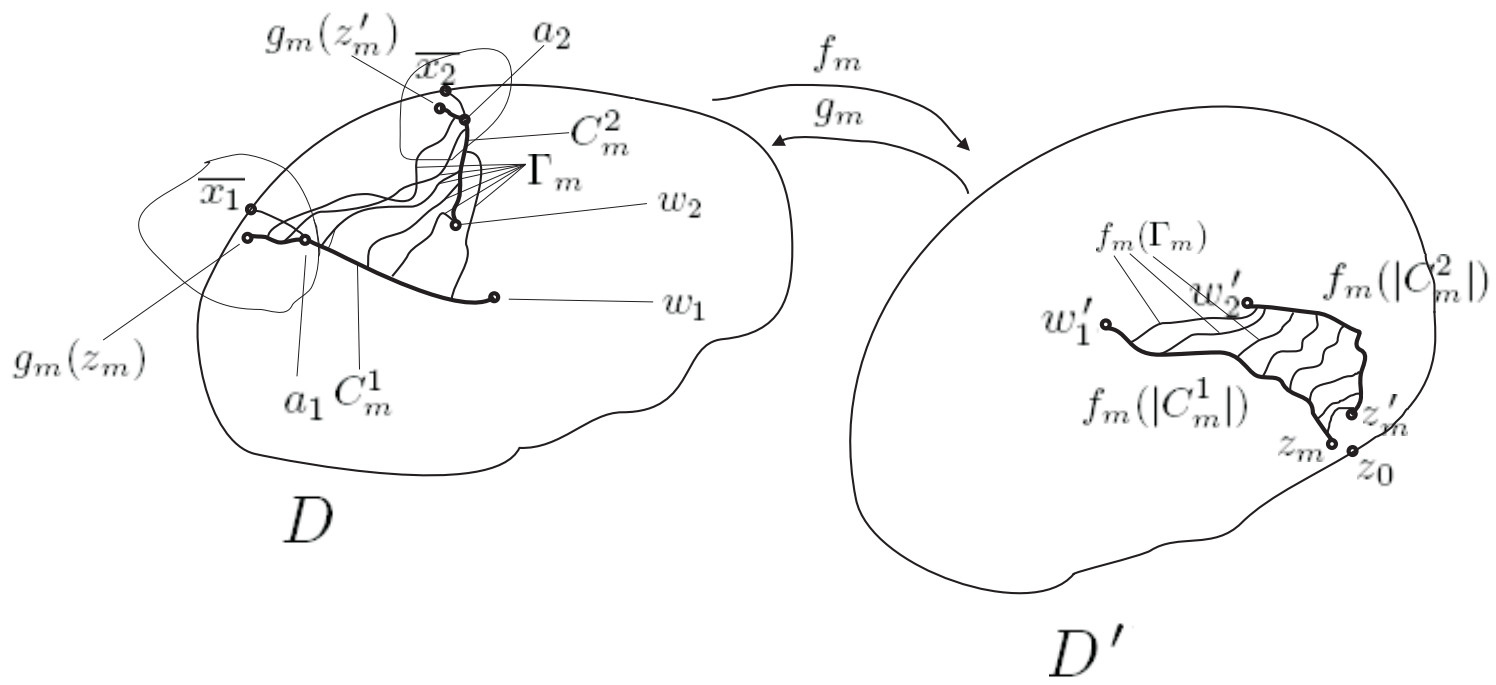

Figure 1. To the proof of Theorem 1

Let $\Gamma_{m}$ be a family of paths connecting $\left|C_{m}^{1}\right|$ and $\left|C_{m}^{2}\right|$ in $D$. Now,

$$
\Gamma_{m}=\bigcup_{i=0}^{N_{0}} \Gamma_{m i}
$$

where $\Gamma_{m i}$ consists of all paths $\gamma:[0,1] \rightarrow D$ with $\gamma(0) \in B\left(x_{i}, l_{0} / 4\right) \cap\left|C_{m}^{1}\right|$ and $\gamma(1) \in\left|C_{2}^{m}\right|$ for $1 \leqslant i \leqslant N_{0}$. Similarly, $\Gamma_{m 0}$ consists of all paths $\gamma:[0,1] \rightarrow D$ with $\gamma(0) \in B\left(\overline{x_{1}}, \delta_{0}\right) \cap\left|C_{m}^{1}\right|$ and $\gamma(1) \in\left|C_{2}^{m}\right|$. By (5) there exists $\sigma_{0}>\delta_{0}>0$ such that

$$
\overline{B\left(\overline{x_{1}}, \sigma_{0}\right)} \cap\left|\gamma_{2}\right|=\varnothing=\overline{U_{2}} \cap\left|\gamma_{1}\right|, \quad \overline{B\left(\overline{x_{1}}, \sigma_{0}\right)} \cap \overline{U_{2}}=\varnothing .
$$

Let $\gamma \in \Gamma_{m 0}$. Since $\gamma(0) \in B\left(\overline{x_{1}}, \delta_{0}\right)$ and $\gamma(1) \in\left|C_{2}^{m}\right|$, by (5) we obtain that $|\gamma| \cap B\left(\overline{x_{1}}, \delta_{0}\right) \neq$ $\varnothing \neq|\gamma| \cap\left(D \backslash B\left(\overline{x_{1}}, \delta_{0}\right)\right)$. Now, by [5, Theorem 1.I, Ch. 5, $\left.\S 46\right]$, there exists $t_{1}^{*} \in(0,1)$ such that $\gamma\left(t_{1}^{*}\right) \in S\left(\overline{x_{1}}, \delta_{0}\right)$. We may assume that $\gamma(t) \in D \backslash \overline{B\left(\overline{x_{1}}, \delta_{0}\right)}$ for $t>t_{1}^{*}$. Similarly, by (7) and [5, Theorem 1.I, Ch. $5, \S 46]$ there exists $t_{2}^{*}>t_{1}^{*}$ with $\gamma\left(t_{2}^{*}\right) \in S\left(\overline{x_{1}}, \sigma_{0}\right)$. We may consider that $\gamma(t) \in D \backslash \overline{B\left(\overline{x_{1}}, \sigma_{0}\right)}$ for $t>t_{2}^{*}$. Thus,

$$
\Gamma_{m 0}>\Gamma\left(S\left(\overline{x_{1}}, \delta_{0}\right), S\left(\overline{x_{1}}, \sigma_{0}\right), A\left(\overline{x_{1}}, \delta_{0}, \sigma_{0}\right)\right) .
$$

Similarly,

$$
\Gamma_{m i}>\Gamma\left(S\left(x_{i}, l_{0} / 4\right), S\left(x_{i}, l_{0} / 2\right), A\left(x_{i}, l_{0} / 4, l_{0} / 2\right)\right) .
$$

Putting

$$
\eta(t)=\left\{\begin{array}{ll}
4 / l_{0}, & t \in\left[l_{0} / 4, l_{0} / 2\right], \\
0, & t \notin\left[l_{0} / 4, l_{0} / 2\right],
\end{array} \quad \eta_{0}(t)= \begin{cases}1 /\left(\sigma_{0}-\delta_{0}\right), & t \in\left[\delta_{0}, \sigma_{0}\right], \\
0, & t \notin\left[\delta_{0}, \sigma_{0}\right],\end{cases}\right.
$$

and $f_{m}:=g_{m}^{-1}$, we obtain by (1) that

$$
\begin{gathered}
M_{\alpha^{\prime}}\left(f_{m}\left(\Gamma\left(S\left(\overline{x_{1}}, \delta_{0}\right), S\left(\overline{x_{1}}, \sigma_{0}\right), A\left(\overline{x_{1}}, \delta_{0}, \sigma_{0}\right)\right)\right)\right) \leqslant\left(1 /\left(\sigma_{0}-\delta_{0}\right)\right)^{\alpha} \cdot\|Q\|_{1}<c_{1}<\infty, \\
M_{\alpha^{\prime}}\left(f_{m}\left(\Gamma\left(S\left(x_{i}, l_{0} / 4\right), S\left(x_{i}, l_{0} / 2\right), A\left(x_{i}, l_{0} / 4, l_{0} / 2\right)\right)\right)\right) \leqslant\left(4 /\left(l_{0}\right)\right)^{\alpha} \cdot\|Q\|_{1}<c_{2}<\infty,
\end{gathered}
$$


where $c_{1}$ and $c_{1}$ are some positive constants, not depending on $m$, and $\|Q\|_{1}=\int_{D} Q(x) d \mu(x)$. We conclude from (6), (8), (9), (10) and subadditivity of modulus that

$$
M_{\alpha^{\prime}}\left(f_{m}\left(\Gamma_{m}\right)\right) \leqslant\left(4 N_{0} / l_{0}^{\alpha}+\left(1 /\left(\sigma_{0}-\delta_{0}\right)\right)^{\alpha}\right)\|Q\|_{1}:=c<\infty .
$$

On the other hand, let $\delta_{1}:=\min \left\{\operatorname{dist}\left(w_{1}^{\prime}, \partial D^{\prime}\right)\right.$, dist $\left.\left(w_{2}^{\prime}, \partial D^{\prime}\right)\right\}$. Now, we obtain that

$$
d^{\prime}\left(f_{m}\left(\left|C_{m}^{1}\right|\right)\right) \geqslant d^{\prime}\left(z_{m}, w_{1}^{\prime}\right) \geqslant \delta_{1} / 2, \quad d^{\prime}\left(f_{m}\left(\left|C_{m}^{2}\right|\right)\right) \geqslant d^{\prime}\left(z_{m}^{\prime}, w_{2}^{\prime}\right) \geqslant \delta_{1} / 2
$$

for some $M_{0} \in \mathbb{N}$ and for all $m \geqslant M_{0}$. Let $U:=B\left(z_{0}, r_{0}\right)=\left\{x \in X^{\prime}: d^{\prime}\left(x, z_{0}\right)<r_{0}\right\}$, where $0<r_{0}<\delta_{1} / 4$ and $\delta_{1}$ is from (12). Notice, that $f_{m}\left(\left|C_{m}^{1}\right|\right) \cap U \neq \varnothing \neq f_{m}\left(\left|C_{m}^{1}\right|\right) \cap\left(D^{\prime} \backslash U\right)$ for sufficiently large $m \in \mathbb{N}$, because $d^{\prime}\left(f_{m}\left(\left|C_{m}^{1}\right|\right)\right) \geqslant \delta_{1} / 2$ and $z_{m} \in f_{m}\left(\left|C_{m}^{1}\right|\right), z_{m} \rightarrow z_{0}$ as $m \rightarrow \infty$. Similarly, $f_{m}\left(\left|C_{m}^{2}\right|\right) \cap U \neq \varnothing \neq f_{m}\left(\left|C_{m}^{2}\right|\right) \cap\left(D^{\prime} \backslash U\right)$. Since $f_{m}\left(\left|C_{m}^{1}\right|\right)$ and $f_{m}\left(\left|C_{m}^{2}\right|\right)$ are continua,

$$
f_{m}\left(\left|C_{m}^{1}\right|\right) \cap \partial U \neq \varnothing, \quad f_{m}\left(\left|C_{m}^{2}\right|\right) \cap \partial U \neq \varnothing,
$$

see, e.g., [5, Theorem 1.I, Ch. $5, \S 46]$. Since $\partial D^{\prime}$ is weakly flat, given $P>0$, there exists a neighborhood $V \subset U$ of $z_{0}$ such that

$$
M_{\alpha^{\prime}}\left(\Gamma\left(E, F, D^{\prime}\right)\right)>P
$$

for any continua $E, F \subset D^{\prime}$ with $E \cap \partial U \neq \varnothing \neq E \cap \partial V$ and $F \cap \partial U \neq \varnothing \neq F \cap \partial V$. Observe that

$$
f_{m}\left(\left|C_{m}^{1}\right|\right) \cap \partial V \neq \varnothing, \quad f_{m}\left(\left|C_{m}^{2}\right|\right) \cap \partial V \neq \varnothing
$$

for sufficiently large $m \in \mathbb{N}$.

Indeed, let $z_{m} \in f_{m}\left(\left|C_{m}^{1}\right|\right), z_{m}^{\prime} \in f_{m}\left(\left|C_{m}^{2}\right|\right)$, where $z_{m}, z_{m}^{\prime} \rightarrow z_{0} \in V$ as $m \rightarrow \infty$. Now, $f_{m}\left(\left|C_{m}^{1}\right|\right) \cap V \neq \varnothing \neq f_{m}\left(\left|C_{m}^{2}\right|\right) \cap V$ for sufficiently large $m \in \mathbb{N}$. In addition, $d^{\prime}(V) \leqslant d^{\prime}(U) \leqslant$ $2 r_{0}<\delta_{1} / 2$. Besides, by (12) we obtain that $d^{\prime}\left(f_{m}\left(\left|C_{m}^{1}\right|\right)\right)>\delta_{1} / 2$. Thus, $f_{m}\left(\left|C_{m}^{1}\right|\right) \cap\left(D^{\prime} \backslash V\right) \neq$ $\varnothing$ and, consequently, $f_{m}\left(\left|C_{m}^{1}\right|\right) \cap \partial V \neq \varnothing$ (see [5, Theorem 1.I, Ch. 5, § 46]). Similarly, $d^{\prime}(V) \leqslant d^{\prime}(U) \leqslant 2 r_{0}<\delta_{1} / 2$. By $(12) d^{\prime}\left(f_{m}\left(\left|C_{m}^{2}\right|\right)\right)>\delta_{1}$, thus $f_{m}\left(\left|C_{m}^{2}\right|\right) \cap\left(D^{\prime} \backslash V\right) \neq \varnothing$. By [5, Theorem 1.I, Ch. 5, $\S 46]$ we have, that $f_{m}\left(\left|C_{m}^{1}\right|\right) \cap \partial V \neq \varnothing$. Thus, (15) is proved.

By (13), (14) and (15), we obtain that $M_{\alpha^{\prime}}\left(f_{m}\left(\Gamma_{m}\right)\right)=M_{\alpha^{\prime}}\left(\Gamma\left(f_{m}\left(\left|C_{m}^{1}\right|\right), f_{m}\left(\left|C_{m}^{2}\right|\right), D^{\prime}\right)\right)>$ $P$, which contradicts (11). The contradiction obtained above disproves the assumption made in (4). The theorem is proved.

Example 1. Consider the case $X=X^{\prime}=\mathbb{R}^{n}, \alpha=\alpha^{\prime}=n, n \geqslant 2$. Let $p \geqslant 1$ be a number, such that $n / p(n-1)<1$. Put $\alpha \in(0, n / p(n-1))$. Let $\mathbb{B}^{n}=\left\{x \in \mathbb{R}^{n}:|x|<1\right\}$. We define a sequence of mappings $f_{m}$ of $\mathbb{B}^{n}$ onto the $B(0,2)$ in the following way:

$$
f_{m}(x)= \begin{cases}\frac{1+|x|^{\alpha}}{|x|} \cdot x, & 1 / m \leqslant|x| \leqslant 1 \\ \frac{1+(1 / m)^{\alpha}}{(1 / m)} \cdot x, & 0<|x|<1 / m\end{cases}
$$

see Figure 2. Notice, that $f_{m}$ satisfies (1) in $\overline{\mathbb{B}^{n}}$ for $Q=\left(\frac{1+|x|^{\alpha}}{\alpha|x|^{\alpha}}\right)^{n-1} \in L^{1}\left(\mathbb{B}^{n}\right)$ at every $x_{0} \in \overline{\mathbb{B}^{n}}$, see [6, proof of Theorem 7]. By [7, Lemma 4.3], $B(0,2)$ has a weakly flat boundary. Observe that $f_{m}$ fixes an infinite number of points of the unit ball for all $m \geqslant 2$.

By Theorem 1 , the family $\mathfrak{G}=\left\{g_{m}\right\}_{m=1}^{\infty}, g_{m}:=f_{m}^{-1}$, is equicontinuous in $\overline{B(0,2)}$. 


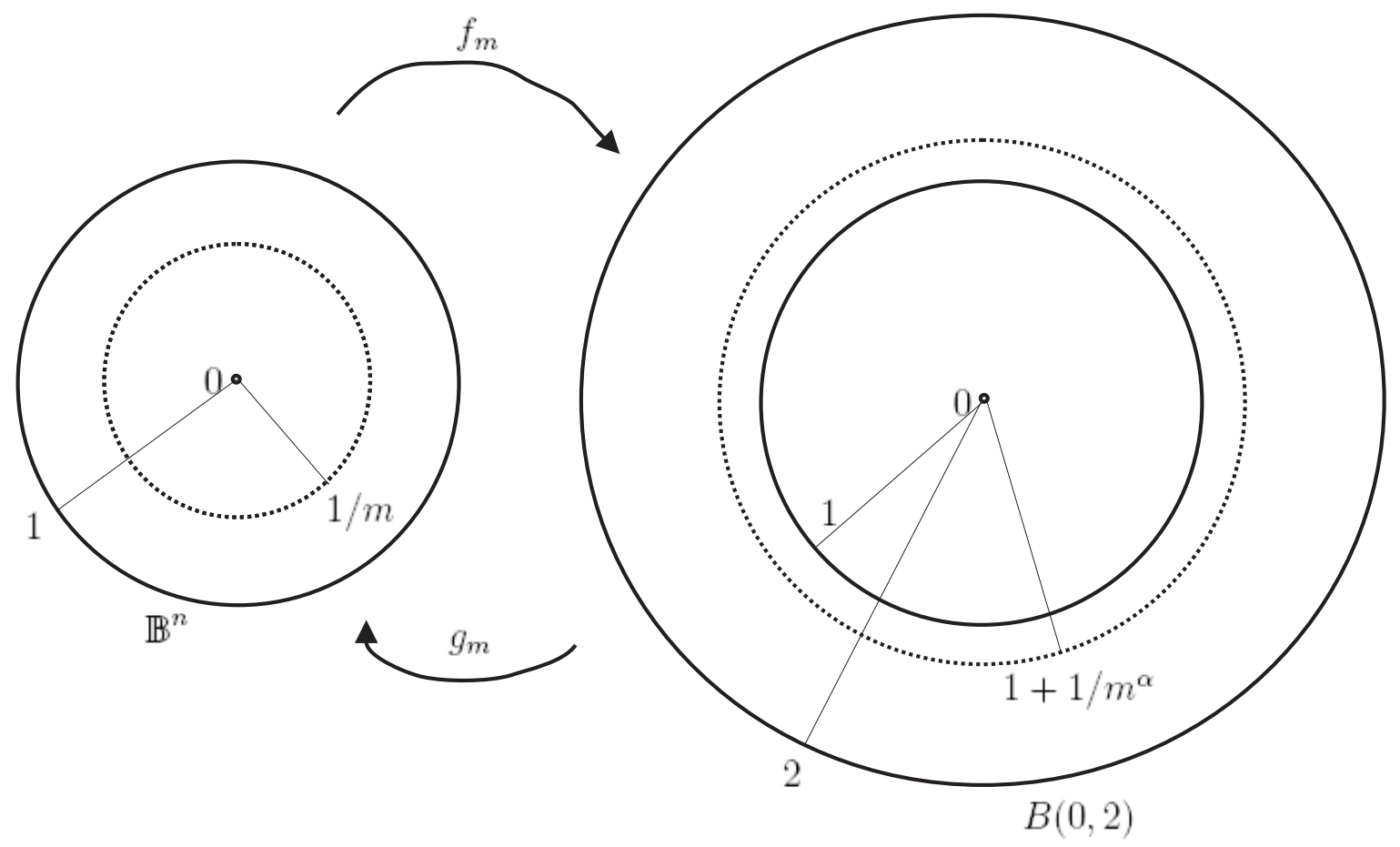

Figure 2. To Example 1

Observe that the "inverse" family $\mathfrak{F}=\left\{f_{m}\right\}_{m=1}^{\infty}$ is not equicontinuous in $\mathbb{B}^{n}$. Indeed, $\left|f_{m}\left(x_{m}\right)-f(0)\right|=1+1 / m^{\alpha} \not \rightarrow 0$ as $m \rightarrow \infty$, where $\left.\left|x_{m}\right|=1 / m\right)$.

The reason for the last circumstance is that $g_{m}$ are not ring $Q$-homeomorphisms with some integrable $Q$ in $B(0,2)$. Let us show this.

Let $K_{I}(x, f)$ be a so-called inner dilatation of $f$ at $x$ (see [6, relation (45)]). By direct calculations, we observe that

$$
\begin{gathered}
g_{m}(y):=f_{m}^{-1}(y)= \begin{cases}\frac{y}{|y|}(|y|-1)^{1 / \alpha}, & 1+1 / m^{\alpha} \leqslant|y|<2, \\
\frac{(1 / m)}{1+(1 / m)^{\alpha}} \cdot y, & 0<|y|<1+1 / m^{\alpha}\end{cases} \\
m=1,2, \ldots, \quad g_{m}: B(0,2) \rightarrow \mathbb{B}^{n} .
\end{gathered}
$$

Arguing similarly to the proof of [6, Theorem 7], we conclude that

$$
K_{I}\left(y, f_{m}\right)= \begin{cases}\frac{|y|}{\alpha(|y|-1)}, & 1+1 / m^{\alpha} \leqslant|y|<2 \\ 1, & 0<|y|<1+1 / m^{\alpha}\end{cases}
$$

Note that $K_{I}\left(y, f_{m}\right) \notin L^{1}(B(0,2))=L^{1}\left(g_{m}\left(\mathbb{B}^{n}\right)\right)$. Indeed, by the Fubini theorem,

$$
\begin{aligned}
& \int_{B(0,2)} K_{I}\left(y, f_{m}\right) d m(y) \geqslant \int_{1<|y|<2} K_{I}\left(y, f_{m}\right) d m(y)= \\
& =\frac{1}{\alpha} \cdot \int_{1<|y|<2} \frac{|y|}{|y|-1} d m(y)=\frac{\omega_{n-1}}{\alpha} \cdot \int_{1}^{2} \frac{r^{n}}{r-1} d r=\infty,
\end{aligned}
$$


where, as usual, $\omega_{n-1}$ denotes the area of the unit sphere $\mathbb{S}^{n-1}$ in $\mathbb{R}^{n}$ and $d m(y)$ is the element of Lebesgue measure. By [8, Statement 1.3], $K_{I}\left(y, f_{m}\right) \leqslant c_{n} \cdot Q(y)$ for almost every $y \in B(0,2)$, where $c_{n}>0$ is some positive constant. Consequently, $Q(y)$ is not integrable, because $K_{I}\left(y, f_{m}\right)$ is not integrable, as well.

\section{REFERENCES}

1. E. A. Sevost'yanov, S. A. Skvortsov, On convergence of mappings in metric spaces with direct and inverse modulus conditions, Ukr. Math. Zh., 70 (2018), №7, 952-967 (in Russian).

2. O. Martio, V. Ryazanov, U. Srebro and E. Yakubov, Moduli in Modern Mapping Theory, Springer Monographs in Mathematics, Springer, New York etc., 2009.

3. V. Ryazanov, R. Salimov, Weakly flat spaces and boundaries in the mapping theory, Ukr. Math. Visnyk, 4 (2007), №2, 199-233 (in Russian); translation in Ukr. Math. Bull., 4 (2007), №2, 199-233.

4. E. S. Smolovaya, Boundary behavior of ring Q-homeomorphisms in metric spaces, Ukr. Mat. Zh., 62 (2010), №5, 682-689 (in Russian); translation in Ukr. Math. Journ., 62 (2010), №5, 785-793.

5. K. Kuratowski, Topology, V.2, Academic Press, New York-London, 1968.

6. E. A. Sevost'yanov, On local and boundary behavior of mappings in metric spaces, Algebra and analiz $\mathbf{2 8}$ (2016), №6, 118-146; translation Local and boundary behavior of maps in metric spaces, St. Petersburg Math. J., 28 (2017), №6, 807-824.

7. M. Vuorinen, On the existence of angular limits of $n$-dimensional quasiconformal mappings, Ark. Mat., 18 (1980), 157-180.

8. E. A. Sevost'yanov, R. R. Salimov, On inner dilatations of the mappings with unbounded characteristic, Ukr. Mat. Visnyk, 8 (2011), №1, 129-143 (in Russian); translation in J. Math. Sci. (N. Y.), 178 (2011), №1, 97-107.

Zhytomyr Ivan Franko State University

Zhytomyr, Ukraine

esevostyanov2009@gmail.com 